\section{Assaying 2-HG's function}

\section{By Kai-Jye Lou, Staff Writer}

Although 2-hydroxyglutarate is a known marker for various cancers, including leukemia, ${ }^{1,2}$ the metabolite's role in the disease remained unclear. Now, U.S. researchers have shown that the compound itself can drive leukemic transformation and that blocking its overproduction with a small molecule from Agios Pharmaceuticals Inc. reversed the effect. ${ }^{3}$

Isocitrate dehydrogenase 1 (IDH1) and IDH2 are critical metabolic enzymes that convert isocitrate to $\alpha$-ketoglutarate, an essential cofactor for a number of enzymes. In 2009, Agios reported that a mutant form of IDH1 commonly found in gliomas gave the enzyme the additional ability to catalyze the formation of the $(R)$ enantiomer of 2-hydroxyglutarate (2-HG) from $\alpha$-ketoglutarate. ${ }^{4,5}$

In 2010, Agios and its academic collaborators reported that acute myelogenous leukemia (AML) cells with mutations in IDH1 and IDH2 also produce $2-$ HG $^{1,2}$

"The Agios group has previously shown that cells with mutations in IDH1 can produce large quantities of 2-HG, though it was still unclear how 2-HG itself was affecting the cells because there was no cellular assay to study the metabolite's function with respect to promoting cancer," said William Kaelin Jr., a professor of medicine at the DanaFarber Cancer Institute and an investigator at the Howard Hughes Medical Institute. "We thought it was important to prove that 2-HG was sufficient to induce leukemic transformation and was not just acting as a biomarker."

To do this, Kaelin's team developed cellular assays to enable the study of 2-HG's role in leukemia. The assays use a human erythroleukemia cell line transduced with lentiviral vectors that encode tagged versions of wild-type or oncogenic mutant IDH1. Cells transduced with oncogenic IDH1 showed greater 2-HG production than those transduced with wild-type $I D H 1$. Moreover, cells transduced with oncogenic IDH1 also acquired two hallmarks of leukemogenesis: growth factor independence and impaired differentiation.

The group then used the assays to show that the $(R)$ enantiomer of 2-HG drove leukemogenesis and that (S)-2-HG did not. A probe of downstream targets of $2-\mathrm{HG}$ that were differentially regulated by the $(R)$ and $(S)$ enantiomers led to the identification of hypoxia-inducible factor prolyl hydroxylase 2 (EGLN1; HIF-PH2; PHD2) as a potential therapeutic target.

Finally, the researchers used a small molecule IDH1 inhibitor research reagent provided by Agios to confirm that inhibiting oncogenic IDH1-induced production of $2-\mathrm{HG}$ reversed leukemogenesis.

The results were published in Science.

Previous studies relied on in vivo phenotypic assays to assess the effects of targeting the IDH1 pathway, which are more labor-intensive and have lower throughput than in vitro cellular assays, said Ross Levine, an associate member in the Human Oncology \& Pathogenesis Program and a physician in the leukemia service at Memorial SloanKettering Cancer Center.

"This study is important from a biological and therapeutic standpoint because one could now test the effect of therapeutic candidates on leukemic transformation," he told SciBX.

Levine added that phenotypic assays only allowed researchers to assess a compound's effects on the growth and proliferation of IDH1mutant cancers. The assays did not show whether a molecule was actually targeting the mechanisms that drive disease.

"These researchers have created a model that will allow us to follow the biology of mutant IDH1," added Katharine Yen, director of biology at Agios. "Before this study, nobody had proven that the 2-HG metabolite itself could be responsible for the downstream oncogenic effects of mutant IDH1."

Yen added that the data also provide additional preclinical evidence to support the development of IDH1 inhibitors in cancer.

Agios has small molecules that inhibit either IDH1 or IDH2 in preclinical development for undisclosed cancer indications. CSO Scott Biller said the company

\section{"We thought it was important to prove that 2-HG was sufficient to induce leukemic transformation and was not just acting as a biomarker." -William Kaelin Jr., Dana-Farber Cancer Institute} hopes to take the compounds into Phase I testing in the next 12-24 months.

\section{New research directions}

The data reported in the paper open up new therapeutic avenues for IDH1-mutant cancers, such as targeting the transformation phenomenon, direct inhibition of 2-HG itself or going after the metabolite's downstream targets.

"The researchers demonstrated that leukemic transformation was reversible, which is critical because it suggests that this transformation is not a one-way phenomenon," said Levine. "The findings suggest one may be able to develop therapeutic compounds that work by reversing leukemic transformation."

Kaelin thinks further probing the activity of the $2-\mathrm{HG}$ enantiomers on downstream targets also could lead to the discovery of new therapeutic targets.

For example, he said, "we looked for proteins that are differentially regulated by the 2-HG enantiomers and came upon EGLN1, which was activated by (R)-2-HG but antagonized by $(S)-2-\mathrm{HG}$."

Kaelin said his group is now trying to translate the in vitro results into in vivo mouse models and trying to determine whether the targets of $(R)-2-$ HG will vary by cancer type. 


\section{ANALYSIS}

"We want to see if the results in leukemia also carry over to other types of cancers known to be driven by mutant $I D H 1$, such as brain tumors and chondrosarcomas," he told SciBX.

The work reported in Science is unpatented. Agios has multiple patent applications filed covering mutant $I D H 1$ as a potential therapeutic target and 2-HG as a potential biomarker.

Celgene Corp. has an exclusive option from Agios to license rights to develop and commercialize an undisclosed number of preclinical compounds targeting cancer metabolism, which includes therapeutics targeting IDH $1 .{ }^{6}$ Celgene's option kicks in after the compounds complete Phase I trials.

Lou, K.-J. SciBX 6(9); doi:10.1038/scibx.2013.205 Published online March 7, 2013

\section{REFERENCES}

1. Gross, S. et al. J. Exp. Med. 207, 339-344 (2010)

2. Ward, P.S. et al. Cancer Cell 17, 225-234 (2010)

3. Losman, J.-A. et al. Science; published online Feb. 7, 2013; doi:10.1126/science.1231677

Contact: William Kaelin Jr., Dana-Farber Cancer Institute, Boston, Mass.

e-mail: william_kaelin@dfci.harvard.edu

4. Dang, L. et al. Nature 462, 739-744 (2009)

5. Lou, K.-J. SciBX 2(48); doi:10.1038/scibx.2009.1749

6. Ward, M. BioCentury 18(18), A7; April 19, 2010

COMPANIES AND INSTITUTIONS MENTIONED

Agios Pharmaceuticals Inc., Cambridge, Mass.

Celgene Corp. (NASDAQ:CELG), Summit, N.J.

Dana-Farber Cancer Institute, Boston, Mass.

Howard Hughes Medical Institute, Chevy Chase, Md.

Memorial Sloan-Kettering Cancer Center, New York, N.Y. 